\title{
Surface Plasmon Lasing Observed in Metal Hole Arrays
}

\author{
Frerik van Beijnum, ${ }^{1}$ Peter J. van Veldhoven, ${ }^{2}$ Erik Jan Geluk,${ }^{2}$ Michiel J. A. de Dood, ${ }^{1}$ \\ Gert W. 't Hooft, ${ }^{1,3}$ and Martin P. van Exter ${ }^{1}$ \\ ${ }^{1}$ Leiden University, Huygens Laboratory, P.O. Box 9504, 2300 RA Leiden, Netherlands \\ ${ }^{2}$ COBRA Research Institute, Technische Universiteit Eindhoven, Postbus 513, 5600 MB Eindhoven, Netherlands \\ ${ }^{3}$ Philips Research Laboratories, Professor Holstlaan 4, 5656 AA Eindhoven, Netherlands
}

(Received 26 February 2013; published 13 May 2013)

\begin{abstract}
Surface plasmons in metal hole arrays have been studied extensively in the context of extraordinary optical transmission, but so far these arrays have not been studied as resonators for surface plasmon lasing at optical frequencies. We experimentally study a metal hole array with a semiconductor (InGaAs) gain layer placed in close $(20 \mathrm{~nm})$ proximity of the metal hole array. As a function of increasing pump power, we observe an intense and spectrally narrow peak, with a clear threshold. This laser emission is donut shaped and radially polarized. Three experimental observations support that the system shows surface plasmon lasing. First, the full wavelength dispersion of the observed resonances can be understood by using a single surface plasmon mode of the system. Second, the polarization of these resonances is as expected for surface plasmons. Third, the magnitude of the avoided crossing, which results from mode coupling at the holes, has a similar magnitude as found in simulations using surface plasmons.
\end{abstract}

DOI: 10.1103/PhysRevLett.110.206802

PACS numbers: 73.20.Mf, 42.25.Fx, 42.55.Px, 42.55.Tv

Surface plasmon lasers and spasers [1] have recently attracted much interest (see, e.g., Ref. [2] for a review) and are attractive candidates for nanoscale lasers. Lasing was reported in different nanoscale resonators: metalcoated nanopillars [3], metal-coated nanorings [4], semiconductor nanowires on a silver film $[5,6]$, and gold nanospheres [7]. Claims of surface plasmon lasing are often hard to substantiate, and experimental observations can be misinterpreted as (surface plasmon) lasing [8]. Therefore, studying surface plasmon lasing in a simple and well known model system will contribute to our understanding of surface plasmon lasing and the limitations thereof.

A metal hole array is a thoroughly studied plasmonic system, mainly in the context of extraordinary optical transmission [9]. Variations on these metal hole arrays are considered to improve the performance [10] of vertical cavity surface emitting lasers [11] and quantum cascade lasers [12,13]. For surface plasmon lasing at optical frequencies, however, metal hole arrays have not yet been considered. Nonetheless, experiments on hole arrays without gain suggest that the holes in the array could provide the required feedback for lasing [9,14-18].

More generally, it was shown that two-dimensional photonic crystals with gain can show laser action [19-21]. Surface plasmons are used in (midinfrared and terahertz) quantum cascade photonic crystal lasers, where the polarization of the surface plasmon conveniently matches that of the optical transition [22-25]. At these wavelengths, however, the absorption loss and the confinement are modest [2], and therefore the quantum cascade lasers are hard to compare with the recently developed nanoscale lasers at optical frequencies [3-7].
In this Letter, we demonstrate surface plasmon lasing in metal hole arrays and substantiate our claim of surface plasmon lasing with three different experimental observations. The experiment, illustrated in Fig. 1(a), studies an optically pumped semiconductor gain layer placed in close proximity of a metal hole array. The luminescence that is transmitted through the hole array is recorded. After discussing this experiment in more detail, we first present the observation of laser action in this system. Thereafter we present angle-, wavelength-, and polarization-resolved measurements on the luminescence which reveal the nature of the laser action.

Experiment.-In Fig. 1(b), we show the layer stack used in our experiment. On a semi-insulating indium phosphide (InP) wafer, a lattice-matched indium gallium arsenide

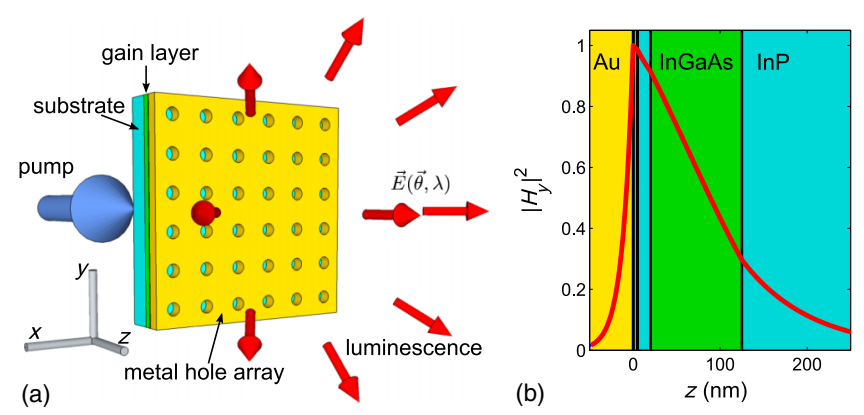

FIG. 1 (color online). (a) Sketch of our experiment: A semiconductor layer, in close proximity of a metal hole array, is pumped optically. We perform an angle-resolved study of the luminescence transmitted through the hole array. (b) The absolute value squared of the magnetic field $H_{y}$ of the designed surface plasmon mode. 
(InGaAs) layer is grown $(105 \mathrm{~nm})$, which is subsequently covered with a thin $(15 \mathrm{~nm})$ layer of InP. Hereafter a $5 \mathrm{~nm}$ protective silicon nitride $(\mathrm{SiN})$ layer is grown by using plasma-enhanced chemical vapor deposition. On these layers we fabricate the metal hole array by depositing $100 \mathrm{~nm}$ gold and $20 \mathrm{~nm}$ chrome on a lithographically defined array of dielectric pillars. To provide sufficient adhesion of the gold onto the SiN, we deposit a very thin (average thickness smaller than $0.5 \mathrm{~nm}$ ) chromium adhesion layer in between these layers. The last step is to etch the pillars away, leaving the subwavelength holes (diameter $160 \mathrm{~nm}$ ).

Using the matrix formulation discussed in Ref. [26], and literature values of the refractive indices, we can numerically derive the guided modes of the layer stack. We present the calculated mode in Fig. 1(b), thereby also illustrating the spatial overlap between the gain layer and the surface plasmon mode. From these calculations we found that a material gain of $\sim 3 \times 10^{3} \mathrm{~cm}^{-1}$ is required to compensate the absorption loss of the surface plasmon. This required gain is large but a realistic number for bulk InGaAs [2,27].

The real part of the calculated effective refractive index is closely related to the wavelength of the resonances in a metal hole array $[17,18]$. For the $105 \mathrm{~nm}$ InGaAs layer the surface plasmon has a calculated effective refractive index of $n_{\text {eff }}=3.43$. The InGaAs layer thickness is chosen to maximize the gain while maintaining single-mode operation. For our system the TE waveguide mode is well below cutoff, as this mode is supported only for InGaAs thicknesses above $\sim 170 \mathrm{~nm}$.

Our experimental setup is as follows. We pump the InGaAs gain layer by using a continuous wave laser at a wavelength of $1064 \mathrm{~nm}$. To pump the structure with a uniform intensity we illuminate a $600 \mu$ m diameter pinhole with the Gaussian laser beam and image this aperture on the sample with a $20 \times$ demagnification. The sample is placed in a helium flow cryostat to study the structure at temperatures down to $5 \mathrm{~K}$. The cryostat has windows on both sides of the hole array, allowing us to pump the structure on one side and study the luminescence at the other side.

We record the luminescence as a function of the angle $\vec{\theta}$, by imaging (magnification $=0.25$ ) the Fourier plane of a microscope objective with a long-working-distance (Mitotoyu, focal distance $10 \mathrm{~mm}$, N.A. $=0.4$ ) on a single-mode fiber placed on a $x y z$ stage. The angular resolution of our setup is approximately $4 \mathrm{mrad}$, which is determined by the modal-field diameter of the single-mode fiber. By scanning the fiber through the Fourier plane and spectrally resolving the fiber output $(\sim 1 \mathrm{~nm}$ resolution), we can create an image of the Fourier plane for each emitted wavelength. In addition to the far-field imaging, we can also adjust the optics such that the fiber is placed in an image plane of the sample, allowing us to make spatial images of the laser for each wavelength.
Laser threshold.-In Fig. 2(a), we plot the recorded spectra as a function of the pump power, at an angle $\left(\theta_{x}, \theta_{y}\right)=(0 \mathrm{mrad}, 48 \mathrm{mrad})$. For most pump powers three maxima in the luminescence are seen. These maxima are associated with different resonances of the hole array of which the origin will be discussed later. As the pump power is increased from 5 (bottom cyan line) to $20 \mathrm{~mW}$ (middle black line), the luminescence maxima at $\sim 1560$ and $\sim 1520 \mathrm{~nm}$ increase in intensity and a third peak becomes visible at $\sim 1480 \mathrm{~nm}$. By increasing the intensity further to $40 \mathrm{~mW}$ (on a second top orange line), the $\sim 1480 \mathrm{~nm}$ peak increases dramatically. A further increase in the pump power to $80 \mathrm{~mW}$ (top red line) shows an increase of the $\sim 1480 \mathrm{~nm}$ peak by more than an order of magnitude, while the intensity of the $1510 \mathrm{~nm}$ peak increases only a factor of 2 . At maximum pump power, the $\sim 1480 \mathrm{~nm}$ peak is 3 orders of magnitude larger than the background luminescence, indicating that the structure is lasing.

The existence of a laser threshold is presented in Fig. 2(b), where we plot the recorded intensity integrated
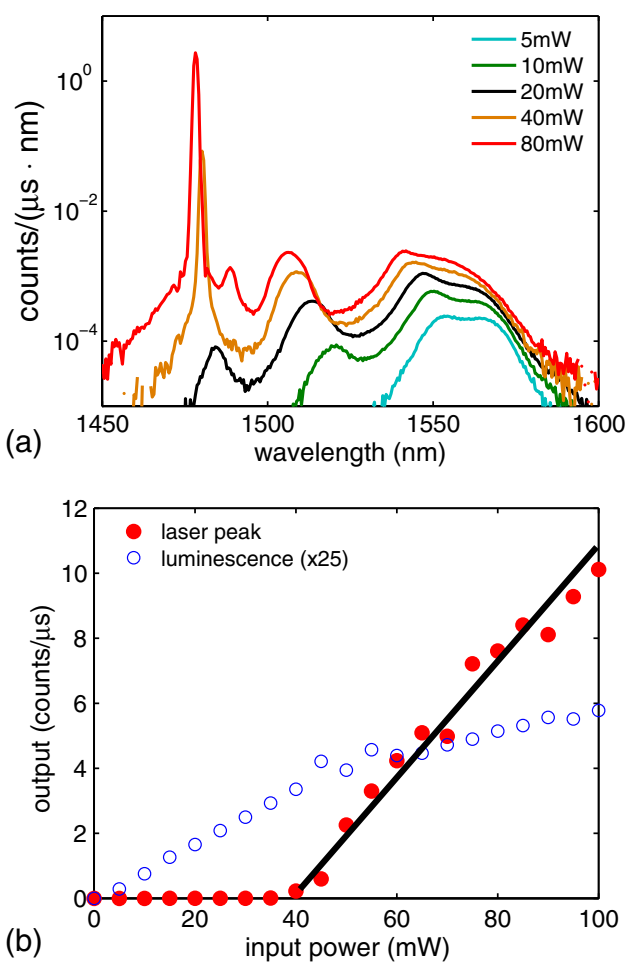

FIG. 2 (color online). (a) Luminescence spectra as a function of pump power, plotted on a semilog scale. For increasing pump power the bandwidth of the luminescence increases until the device starts lasing. Above threshold, the emission of the nonlasing resonances starts to saturate at a maximum intensity ( $80 \mathrm{~mW}$ corresponds to $\sim 11 \mathrm{~kW} / \mathrm{cm}^{2}$ ). (b) The output in the lasing peak and in the luminescence in the range of 1485-1600 nm. The power in the lasing peak shows a clear threshold (solid red dots). The black line is a guide to the eye. The luminescence outside the lasing peak starts to level off, as expected for lasing in semiconductor devices (open blue circles). 
over the spectral peak at $\sim 1480 \mathrm{~nm}$ as a function of input power. A distinct laser threshold is seen at roughly $40 \mathrm{~mW}$ pump power. We also plot the integrated luminescent intensity in the wavelength range of 1485-1600 nm. The integrated luminescence first increases linearly with the input power, but for powers larger than the threshold it starts to level off, indicating the expected carrier pinning [3,28].

We observe lasing in our sample up to a temperature of $\sim 150 \mathrm{~K}$. At higher temperatures the maximal attainable gain of the semiconductor is too low to compensate the losses. However, we expect that further engineering of the layer stack shown in Fig. 1(b) can reduce the gain requirement by roughly a factor of 2 . This could allow room temperature operation, as previously realized by using InGaAs in a metallic nanocavity [29].

The results presented in Fig. 2 are for a laser that has a lattice spacing of $470 \mathrm{~nm}$ and lases at $1478 \mathrm{~nm}$. We also fabricated lasers with a lattice parameter of 460 and $450 \mathrm{~nm}$. Both devices show lasing, too, with the laser wavelengths of 1450 and $1419 \mathrm{~nm}$, respectively. Hence the ratio between the laser wavelength and lattice parameter is a constant, namely, $3.15 \pm 0.01$. This clear relation between the lattice constant and the laser wavelength shows that the hole array is used as a resonator.

Dispersion.-To reveal the origin of the three resonant peaks in Fig. 2(a), we will now study the wavelength dispersion of these resonances. The emission angle as a function of wavelength can often be understood by using a simple model that assumes uncoupled traveling waves. For two neighboring holes on either the $x$ axis or $y$ axis, this traveling wave has a phase difference of $k_{x} a_{0}$ or $k_{y} a_{0}$, respectively, with $\vec{k}=\left(k_{x}, k_{y}\right)$ the wave vector of the traveling wave. This linear phase results in a plane wave emitted at an angle $\left(\theta_{x}, \theta_{y}\right)$. By relating $k_{x}$ and $k_{y}$ to the total momentum, $k^{2}=k_{x}^{2}+k_{y}^{2}$, the emission angle can be calculated [14]:

$$
n_{\mathrm{eff}}^{2} k_{0}^{2}=|k|^{2}=\left|k_{\|, x}-m_{x} G\right|^{2}+\left|k_{\|, y}-m_{y} G\right|^{2}
$$

with $G=2 \pi / a_{0}, n_{\text {eff }}$ the mode index of the traveling wave, $\left(m_{x}, m_{y}\right)$ the diffraction order, $k_{\|, x}=k_{0} \sin \theta_{x}$, and $k_{\|, y}=k_{0} \sin \theta_{y}$. Equation (1) describes a circle of radius $k$ around the point $\left(m_{x} G, m_{y} G\right)$. In our measurements, the mode index is the only unknown parameter. Importantly, Eq. (1) describes uncoupled traveling waves but often suffices to understand the main features of the dispersion $[9,14]$. For the relatively weakly interacting surface plasmons, the avoided crossing can be observed only when curves of different $\left(m_{x}, m_{y}\right)$ intersect.

In Fig. 3, we show the measured angle-resolved luminescence for eight wavelengths when the laser is operated below threshold, along with a plot of Eq. (1) for $n_{\text {eff }}=$ 3.26. We plot the images with decreasing wavelength, i.e., increasing $k$. The general behavior is well predicted by the simple model and shows the square symmetry of

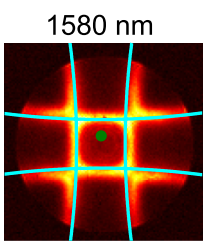

$1500 \mathrm{~nm}$

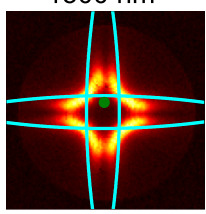

$1560 \mathrm{~nm}$

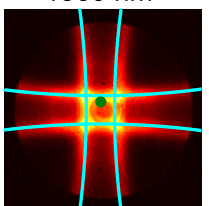

$1480 \mathrm{~nm}$

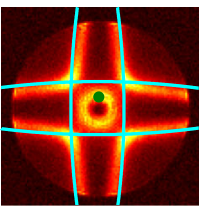

$1540 \mathrm{~nm}$

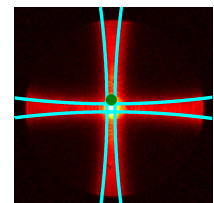

$1460 \mathrm{~nm}$

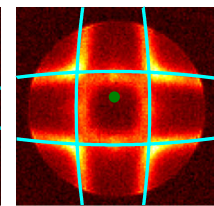

$1520 \mathrm{~nm}$

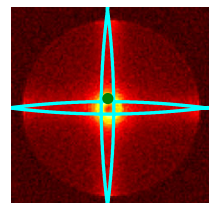

$1440 \mathrm{~nm}$

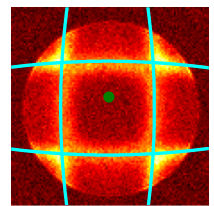

FIG. 3 (color online). Far-field emission patterns at eight wavelengths, observed below laser threshold. These images reveal the dispersion of the modes inside the metal hole array. The cyan lines correspond to the dispersion of uncoupled traveling waves with an effective index of 3.26 , showing that only one mode is needed to understand the dispersion of the luminescence maxima. The circle shows the numerical aperture of the microscope objective (N.A. $=0.4$ ), and the optical axis is the center of this circle. The green dot indicates the angle where the measurements in Fig. 2 are performed.

the lattice. As a function of $k$ the circles increase in radius and, thus, move towards the optical axis (1580-1540 nm), cross it (1540-1520 nm), and thereafter move away from it (1520-1440 nm). At the angular position where the theoretical lines intersect, coupling is observed (1520-1440 nm), changing the far field drastically. From the correspondence between the figures and the theory for uncoupled modes, we conclude that one value of $n_{\text {eff }}$ is required to understand all far-field luminescence patterns. Given that our layer stack supports only a single mode, the surface plasmon, this is the first experimental observation that supports our claim of surface plasmon lasing.

The value of $n_{\text {eff }}$ found is distinctly larger than the refractive index of the substrate $\left(n_{\mathrm{InP}}=3.08\right)$. Therefore, the luminescence seen in Fig. 3 is not associated with the emergence of a new diffraction order. The found value of $n_{\text {eff }} \sim 3.26$ is low compared to the value we expect for the surface plasmon mode (3.43). This difference is partially due to cooling the sample and a carrier-induced refractive index change. Furthermore, the predicted value of $n_{\text {eff }}$ is very sensitive to the thickness of the low-index layer of $\mathrm{SiN}$. Increasing the thickness of this layer by $10 \mathrm{~nm}$ already lowers the index of the surface plasmon from 3.43 to the experimentally observed value of 3.26. An alternative explanation for the low value of $n_{\text {eff }}$ is a thin layer of oxide on the InP.

The luminescence in Fig. 3 is recorded without using a polarizer. By studying the dispersion of the resonances for $p$ and $s$ polarization separately, we can show whether the observed resonances are mediated via a TE or a TM mode [30]. Performing this measurement, we find three $p$-polarized resonances and one $s$-polarized resonance if 
we scan along $\theta_{y}$ while setting $\theta_{x}=0$ (see Supplemental Material [31]). The observed polarization dependence of the resonances is consistent with a TM mode around $\lambda / a_{0}=n_{\text {eff }}$, providing the second observation that substantiates our claim of surface plasmon lasing.

From this dispersion we can also quantify the magnitude of the coupling that the mode experiences from the holes. As discussed in the Supplemental Material [31], the avoided crossing has a splitting of roughly $65 \mathrm{~nm}$, which compares very well with the simulated avoided crossing for surface plasmons $(70 \mathrm{~nm})$, while the splitting for a TE mode is less than $1 \mathrm{~nm}$. Hence, the magnitude of the splitting of the avoided crossing is the third experimental observation that supports our claim of surface plasmon lasing.

Because all luminescence shown in Figs. 2 and 3 is related to a surface plasmon mode at the metalsemiconductor interface, the three resonant peak in Fig. 2 can now be interpreted as follows. In several studies it is shown that the coupling induced by the holes leads to resonances with different frequencies and loss rates $[17,30,32,33]$. A low loss resonance is commonly explained by the notion that the associated field is smaller at the holes and therefore the resonance experiences lower outcoupling loss [30]. This behavior of the field is demonstrated for surface plasmons [32] and calculations on socalled band edge lasers [34,35]. The lasing mode in our structure is the resonance that is expected to have the smallest loss rate.

Polarization of laser emission.-Last, we found that the laser emission is donut shaped, as shown Fig. 4(a). This behavior compares well with transmission experiments and simulations on these hole arrays, where it was shown that this resonance vanishes when excited at normal incidence $[17,32]$. The diameter of the ring we observe is $120 \mathrm{mrad}$, which is Fourier related to a diameter of roughly $16 \mu \mathrm{m}$ on the sample. The donut-shaped emission reproduces in all lasing samples, although the beam quality varies from sample to sample.

To study the local polarization of the lasing mode, we show an image of the emission when the vertical polarization is selected by using a polarizer [Fig. 4(b)]. This image, on the same intensity scale as Fig. 4(a), now shows two lobes instead of a ring. If we set the polarizer at an arbitrary angle of $30^{\circ}$ the lobes rotate along with the polarizer, showing that the laser emission is radially polarized. This radial polarization is consistent with the polarization analysis performed in the Supplemental Material [31], which shows that for angles $\left(0, \theta_{y}\right)$ the polarization is vertical and for angles $\left(\theta_{x}, 0\right)$ the polarization is horizontal.

In Fig. 4(c), we also show an real-space image of the laser mode. The round circular spot is comparable to the size of the pumped area $(\sim 30 \mu \mathrm{m})$, albeit somewhat larger $(\sim 40 \mu \mathrm{m})$. This difference in size is probably due
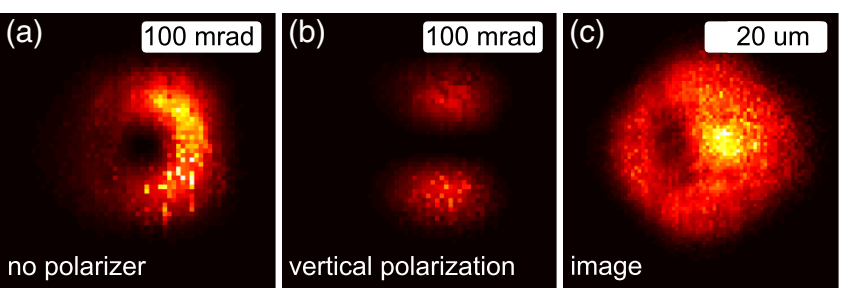

FIG. 4 (color online). (a), (b) Angle-resolved intensity at the lasing wavelength, above threshold at $5 \mathrm{~K}$. (a) No analyzing polarizer. (b) Vertical polarization selected. (c) Direct image of the luminescence at the lasing wavelength. The structure lases at all positions where it is pumped.

to carrier diffusion, which is expected to be a few micrometers. The observed emission is laser light, as a similar image at a nonlasing wavelength is 3 orders of magnitude less intense. When comparing Figs. 4(a) and 4(c), we note that the far-field spot is not Fourier related to the image of the lasing spot. This suggests that the laser has multiple spatial modes, a hypothesis that is supported by the observation that we can reduce the size of the pump spot and still obtain lasing.

Conclusion.-We have demonstrated surface plasmon lasing in metal hole arrays. The luminescent input-output characteristic shows clear threshold behavior and the integrated emission levels off, which indicates carrier density pinning $[3,28]$. The laser emission is not linearly polarized but radially polarized and donut shaped. We find three experimental observations that support our claim of surface plasmon lasing: First, the angle-resolved luminescence shows that all observed resonances are mediated via one mode, while the layer stack only supports a surface plasmon; second, the polarization of the four observed resonances is as expected for surface plasmons; third, the dispersion shows a large avoided crossing of which the magnitude is almost the same as found from simulations using surface plasmons.

Compared to the recently reported nanoscale lasers [3-7], our surface plasmon laser has three distinct advantages: first, using the far-field luminescence we can reveal the band structure of the metal hole array; second, because our laser produces a compact beam, we can analyze its shape and polarization; third, the resonator is thoroughly studied and can be understood by using relatively simple modeling. All three benefits are important when one is trying to understand surface plasmon lasing and its limitations. Further miniaturization of this laser, for example, by increasing the feedback provided by the holes, would make this laser interesting for applications like photonic integration.

We acknowledge M. T. Hill, R. Lenssen, W. Löffler, M. J.H. Marell, E. Smalbrugge, and T. de Vries for discussions. This work is part of the research program of the Foundation for Fundamental Research on Matter (FOM), which is part of the Netherlands Organisation for Scientific Research (NWO). 
[1] D. J. Bergman and M. I. Stockman, Phys. Rev. Lett. 90, 027402 (2003).

[2] P. Berini and I. De Leon, Nat. Photonics 6, 16 (2011).

[3] M. T. Hill, Y.-S. Oei, B. Smalbrugge, Y. Zhu, T. de Vries, P. J. van Veldhoven, F. W. M. van Otten, T. J. Eijkemans, J.P. Turkiewicz, H. de Waardt, E. J. Geluk, S.-H. Kwon, Y.-H. Lee, R. Notzel, and M. K. Smit, Nat. Photonics 1, 589 (2007).

[4] M. W. Kim and P. C. Ku, Appl. Phys. Lett. 98, 201105 (2011).

[5] R. F. Oulton, V. J. Sorger, T. Zentgraf, R.-M. Ma, C. Gladden, L. Dai, G. Bartal, and X. Zhang, Nature (London) 461, 629 (2009).

[6] Y.-J. Lu, J. Kim, H.-Y. Chen, C. Wu, N. Dabidian, C. E. Sanders, C.-Y. Wang, M.-Y. Lu, B.-H. Li, X. Qiu, W.-H. Chang, L.-J. Chen, G. Shvets, C.-K. Shih, and S. Gwo, Science 337, 450 (2012).

[7] M. A. Noginov, G. Zhu, A. M. Belgrave, R. Bakker, V. M. Shalaev, E. E. Narimanov, S. Stout, E. Herz, T. Suteewong, and U. Wiesner, Nature (London) 460, 1110 (2009).

[8] M. C. Gather, Nat. Photonics 6, 708 (2012).

[9] T. W. Ebbesen, H. J. Lezec, H. F. Ghaemi, T. Thio, and P. A. Wolff, Nature (London) 391, 667 (1998).

[10] H. J. Lezec, A. Degiron, E. Devaux, R. A. Linke, L. Martin-Moreno, F. J. Garcia-Vidal, and T. W. Ebbesen, Science 297, 820 (2002).

[11] S. Shinada, J. Hashizume, and F. Koyama, Appl. Phys. Lett. 83, 836 (2003).

[12] N. Yu, R. Blanchard, J. Fan, F. Capasso, T. Edamura, M. Yamanishi, and H. Kan, Appl. Phys. Lett. 93, 181101 (2008).

[13] N. Yu, Q. Wang, and F. Capasso, Laser Photonics Rev. 6, 24 (2012).

[14] W. L. Barnes, W. A. Murray, J. Dintinger, E. Devaux, and T. W. Ebbesen, Phys. Rev. Lett. 92, 107401 (2004).

[15] L. Pang, K. A. Tetz, and Y. Fainman, Appl. Phys. Lett. 90, 111103 (2007).

[16] D. Stolwijk, E. F.C. Driessen, M. A. Verschuuren, G. W. 't Hooft, M.P. van Exter, and M.J.A. de Dood, Opt. Lett. 33, 363 (2008).

[17] H. T. Liu and P. Lalanne, Nature (London) 452, 728 (2008).

[18] F. van Beijnum, C. Rétif, C. B. Smiet, H. Liu, P. Lalanne, and M. P. van Exter, Nature (London) 492, 411 (2012).
[19] M. Meier, A. Mekis, A. Dodabalapur, A. Timko, R.E. Slusher, J. D. Joannopoulos, and O. Nalamasu, Appl. Phys. Lett. 74, 7 (1999).

[20] E. Miyai, K. Sakai, T. Okano, W. Kunishi, D. Ohnishi, and S. Noda, Nature (London) 441, 946 (2006).

[21] H. Matsubara, S. Yoshimoto, H. Saito, Y. Jianglin, Y. Tanaka, and S. Noda, Science 319, 445 (2008).

[22] R. Colombelli, K. Srinivasan, M. Troccoli, O. Painter, C. F. Gmachl, D. M. Tennant, A. M. Sergent, D. L. Sivco, A. Y. Cho, and F. Capasso, Science 302, 1374 (2003).

[23] G. Scalari, L. Sirigu, R. Terazzi, C. Walther, M. I. Amanti, M. Giovannini, N. Hoyler, J. Faist, M. L. Sadowski, H. Beere, D. Ritchie, L. A. Dunbar, and R. Houdré, J. Appl. Phys. 101, 081726 (2007).

[24] L. Sirigu, R. Terazzi, M. I. Amanti, M. Giovannini, J. Faist, L.A. Dunbar, and R. Houdré, Opt. Express 16, 5206 (2008).

[25] Y. Chassagneux, R. Colombelli, W. Maineult, S. Barbieri, H.E. Beere, D. A. Ritchie, S. P. Khanna, E. H. Linfield, and A. G. Davies, Nature (London) 457, 174 (2009).

[26] P. Yeh, Optical Waves in Layered Media (Wiley, New York, 1998).

[27] M. T. Hill, J. Opt. Soc. Am. B 27, B36 (2010).

[28] R. E. Slusher, A. F. J. Levi, U. Mohideen, S. L. McCall, S. J. Pearton, and R. A. Logan, Appl. Phys. Lett. 63, 1310 (1993).

[29] M. T. Hill, M. Marell, E. S. P. Leong, B. Smalbrugge, Y. Zhu, M. Sun, P. J. van Veldhoven, E. J. Geluk, F. Karouta, Y.-S. Oei, R. Nötzel, C.-Z. Ning, and M. K. Smit, Opt. Express 17, 11107 (2009).

[30] P. Paddon and J. F. Young, Phys. Rev. B 61, 2090 (2000).

[31] See Supplemental Material at http://link.aps.org/ supplemental/10.1103/PhysRevLett.110.206802 for measurements of the luminescence and simulations of the transmission of $s$ and $p$ polarization.

[32] C. Ropers, D. J. Park, G. Stibenz, G. Steinmeyer, J. Kim, D. S. Kim, and C. Lienau, Phys. Rev. Lett. 94, 113901 (2005).

[33] M. J. A. de Dood, E. F. C. Driessen, D. Stolwijk, and M. P. van Exter, Phys. Rev. B 77, 115437 (2008).

[34] S. Noda, M. Yokoyama, M. Imada, A. Chutinan, and M. Mochizuki, Science 293, 1123 (2001).

[35] G. A. Turnbull, P. Andrew, M. J. Jory, W. L. Barnes, and I. D. W. Samuel, Phys. Rev. B 64, 125122 (2001). 